\title{
Saúde masculina: tão negligenciada, principalmente pelos homens
}

Ricardo M. R. Meirelles ${ }^{1}$, Alexandre Hohl ${ }^{2}$

E mbora a saúde da mulher, nas suas especificidades, tenha sido objeto de atenção Cdesde os anos 70 do século $\mathrm{XX}$, ações mais abrangentes em relação à saúde masculina, além dos cuidados com a próstata e o câncer de testículo, só se tornaram mais consistentes no século XXI. Isso apesar de as evidências mostrarem que os homens morrem mais do que as mulheres em todas as faixas etárias, inclusive antes do nascimento, pois a maioria dos abortos espontâneos de fetos sem malformações é do sexo masculino (1). Na própria história da Sociedade Brasileira de Endocrinologia e Metabologia, observa-se que o Departamento de Endocrinologia Feminina foi criado anos antes de se anexar a ele a Andrologia. A testosterona injetável só surgiu no mercado em 1954 (2), cerca de 20 anos após a indústria farmacêutica ter produzido o benzoato de estradiol parenteral e 12 anos depois dos comprimidos de estrogênios conjugados (3). Só recentemente surgiram trabalhos científicos com metodologia confiável que demonstraram que a deficiência de testosterona, além da reconhecida influência na diminuição de libido, também se associa à síndrome metabólica, com suas consequências cardiovasculares, e que a recuperação de níveis fisiológicos desse hormônio é capaz de melhorar a insuficiência cardíaca (4) e os sintomas anginosos (5).

Ainda são poucos os centros endocrinológicos brasileiros que desenvolvem trabalhos relacionados à saúde masculina, o que representou uma grande dificuldade para os editores deste fascículo obterem um número razoável de trabalhos merecedores de figurarem nesta primeira edição dos Arquivos dedicada à Andrologia (o fascículo sobre Endocrinologia Feminina foi publicado há mais de oito anos, em agosto de 2001). Consideramos importante a produção deste exemplar, não só para divulgar o trabalho dos colegas que militam nesta área, mas também para estimular novas linhas de pesquisa e o aumento do número de Serviços de Endocrinologia que as incluam entre os seus objetivos.

Como definir saúde masculina? Um problema de saúde masculina seria aquele, proveniente de fatores fisiológicos, psicológicos, sociais, culturais ou ambientais, que tem um impacto específico sobre meninos ou homens e/ou em que são necessárias intervenções específicas, para meninos ou homens, de modo a obter melhoras na saúde e no bem-estar, seja no âmbito individual ou populacional, segundo o Men's Health Forum (6). No campo da Endocrinologia, inúmeras situações se enquadram nessa definição. Este número dos Arquivos traz trabalhos que abordam vários desses problemas, incluindo a relação entre acromegalia, IGF-l e câncer de próstata, a influência da testosterona na composição corporal e sua utilidade no tratamento da obesidade e do diabetes melito em homens hipogonádicos, ação dos androgênios na densidade mineral óssea, o hipogonadismo associado ao traumatismo cranioencefálico e a influência dos estrogênios e androgênios sobre a próstata em roedores e humanos.

O hipogonadismo do envelhecimento masculino merece atenção especial. Diferentemente do que ocorre na mulher, o declínio dos níveis de testosterona é gradual, nem
1 Professor-associado de Endocrinologia da Pontifícia Universidade Católica (PUC-Rio de Janeiro); presidente da Sociedade Brasileira de Endocrinologia e Metabologia (SBEM); diretor do Instituto Estadual de Diabetes e Endocrinologia Luiz Capriglione (IEDE), Rio de Janeiro, RJ, Brasil 2 Presidente da Sociedade Brasileira de Endocrinologia e Metabologia regional Santa Catarina (SBEM$\mathrm{SC})$; diretor do Departamento de Endocrinologia Feminina e Andrologia (DEFA) da SBEM; mestre em Neurociências pela Universidade Federal de Santa Catarina (UFSC); professor de Endocrinologia da UFSC; médico endocrinologista do Núcleo de Pesquisas em Neurologia Experimental e Clínica (NUPNEC), UFSC, Florianópolis, SC, Brasil

Correspondência para: Ricardo M. R. Meirelles Rua Visconde de Pirajá, 330/913 - Ipanema

22410-001 - Rio de Janeiro, RJ Brasil

rmeirelles@openlink.com.br 
sempre atinge limites capazes de gerar consequências clínicas e, mesmo quando provoca sintomas, estes são inespecíficos e podem não ensejar a dosagem de testosterona que permitiria o diagnóstico. A associação com outras patologias, como a obesidade, a depressão e o diabetes melito, pode dificultar ainda mais a suspeita de hipogonadismo, sendo seus sintomas atribuídos à doença associada, cujo próprio tratamento poderia se beneficiar da normalização dos níveis de androgênios. Por outro lado, de forma semelhante ao que ocorreu com a terapia hormonal da menopausa, em relação ao câncer de mama, o uso terapêutico de testosterona ainda suscita em muitos o medo de que possa causar câncer de próstata, embora não haja amparo científico para esse temor (7). Na verdade, os casos mais agressivos de câncer de próstata parecem se associar a níveis baixos de testosterona (8).

Homens não gostam de ir ao médico e, com exceção dos urologistas, todos os outros especialistas atendem um número muito maior de mulheres do que de homens. Essa negligência com a própria saúde tem raízes culturais e a relutância muitas vezes está associada à ideia de que admitir a possibilidade de doença é uma "fraqueza" incompatível com a masculinidade. Isso faz com que as doenças nos homens sejam diagnosticadas mais tardiamente, acarretando pior prognóstico e encurtando a expectativa de vida. Os serviços de saúde são considerados, pelos homens, como destinados às mulheres e às crianças, uma vez que a maioria das campanhas sanitárias é voltada às peculiaridades dessa clientela.
As políticas públicas de saúde só agora estão reconhecendo a necessidade de oferecer programas voltados para a prevenção e o tratamento das patologias mais prevalentes ou específicas dos homens. Mais do que criar a infraestrutura apropriada, será preciso mudar o comportamento da população masculina com relação à própria saúde. Essa é uma tarefa que compete a todos nós.

\section{REFERÊNCIAS}

1. Byrne J, Warburton D, Opitz JM, Reynolds JF. Male excess among anatomically normal fetuses in spontaneous abortions. Am J Med Genet. 1987;26:605-11.

2. Nieschlag E, Behre HM, Bouchard P, Corrales JJ, JonesTH, Stalla GK, et al. Testosterone replacement therapy: current trends and future directions. Hum Reprod Update. 2004;10:409-19.

3. Stefanick ML. Estrogens and progestins: background and history, trends in use, and guidelines and regimens approved by the US Food and Drug Administration. Am J Med. 2005;118 Suppl 12B:64-73.

4. Caminiti G, Volterrani M, lellamo F, Marazzi G, Massaro R, Miceli $M$, et al. Effect of long-acting testosterone treatment on functional exercise capacity, skeletal muscle performance, insulin resistance, and baroreflex sensitivity in elderly patients with chronic heart failure a double-blind, placebo-controlled, randomized study. J Am Coll Cardiol. 2009;54:919-27.

5. Malkin CJ, Pugh PJ, Morris PD, Kerry KE, Jones RD, Jones TH, et al. Testosterone replacement in hypogonadal men with angina improves ischaemic threshold and quality of life. Heart. 2004;90:871-6.

6. Wilkins D, Baker P. Getting it sorted: a policy programme for men's health. London: The Men's Health Forum; 2004.

7. Morgentaler A. Testosterone and prostate cancer: an historical perspective on a modern myth. Eur Urol. 2006;50:935-9.

8. Severi G, Morris HA, Maclnnis RJ, English DR, Tilley W, Hopper $\mathrm{JL}$, et al. Circulating steroid hormones and the risk of prostate cancer. Cancer Epidemiol Biomarkers Prev. 2006;15:86-91. 